\title{
Análisis del crédito comercial en pequeñas y medianas empresas de derivados lácteos
}

\author{
Boscán, Mariby* \\ Sandrea, Maryana*
}

\section{Resumen}

Las empresas de reducido tamaño, frecuentemente enfrentan grandes retos en el entorno económico y social, que requieren estrategias cada vez más eficientes para el uso de los recursos financieros de que disponen. Por tanto, en el presente artículo se pretende analizar la utilización del crédito comercial como instrumento de financiamiento para las empresas productoras de derivados lácteos del estado Zulia, identificando adicionalmente sus necesidades de financiamiento. La investigación es descriptiva y de campo. Entre los resultados destacan: El crédito comercial figura como el principal instrumento de financiación a corto plazo $(75 \%)$, siendo el plazo para la cancelación el criterio mayormente considerado. Al analizar las necesidades de financiamiento, se encontró que el monto requerido supera los cincuenta millones de Bolívares; el período debería ser entre 1 y 3 años y la tasa de interés podría oscilar entre $10 \%$ y $20 \%$. Se concluye, que es necesario, coordinar el horizonte temporal del crédito con su uso y evaluar las tasas de interés versus la rentabilidad de la inversión.

Palabras clave: Crédito comercial, fuentes de financiamiento, pequeñas y medianas empresas, sector de derivados lácteos.

\section{Analysis of Commercial Credit in Small and Medium Enterprises Producing Dairy Products}

\section{Abstract}

Small businesses frequently face great challenges in the economic and social surroundings and increasingly require efficient strategies for using the financial resources they have. This article analyzes the use of commercial credit as a financing instrument for dairy product companies in the State of Zulia, identifying furthermore, their financing needs. The research is of a descriptive, field

\section{Recibido: 05-12-07 Aceptado: 03-03-09}

Magíster en Gerencia de Empresas, Economistas. Profesoras agregadas e Investigadoras de la Universidad del Zulia. Instituto de Investigaciones de la Facultad de Ciencias Económicas y Sociales. Departamento de Estudios de Microeconomía. Email: marysandrea@yahoo.com y maribyboscán@yahoo.com. 
type. Among the results are: commercial credit appears to be the main instrument for short-term financing $(75 \%)$, with the deadline for repayment as the most-considered criterion. When analyzing financing needs, it was found that the amount requested exceeds fifty million Bolivars; the period should be between 1 and 3 years and the interest rate could range between $10 \%$ and $20 \%$. Conclusions were that it is necessary to coordinate the temporal credit horizon with its use and assess interest rates versus the return on the investment.

Key words: Commercial credit, financing sources, small and medium enterprises, dairy products sector.

\section{Introducción}

En los últimos años ha tomado gran auge el estudio de la Pequeña y Mediana Empresa (PYME) y su impacto en el desarrollo nacional, regional y local, con lo cual se ha abordado su comprensión desde distintas ópticas, que tienen como eje de análisis la eficiencia de la PYME dado la flexibilidad de su proceso productivo para adaptarse a los cambios del entorno, su capacidad de generación de puestos de trabajo, las políticas para su desarrollo, entre otras.

Las Pequeñas y Medianas Empresas, constantemente son objeto de estrategias de desarrollo, enfrentando exigencias del entorno y tecnologías gerenciales, que requieren cada vez más un buen manejo de los recursos de que disponen, especialmente los financieros, así como también de la planificación, mercadeo, entre otros, que les permitan desarrollar ventajas para afrontar exitosamente la realidad de la cual forman parte (IESA, 1997).

Algunas PYME se han convertido en precursoras de nuevas formas de organización industrial. El papel tradicional de estos establecimientos como productores de bienes y servicios con bajo contenido tecnológico y alta utilización de mano de obra se ha modificado, tal como lo evidencia el surgimiento de pequeñas y medianas empresas innovadoras. No se trata de una tendencia generalizada, pero en algunos países y ciertas ramas industriales funcionan con capacidad para incorporar tecnologías productivas y avanzadas, emplear trabajadores calificados y realizar innovaciones tanto en los productos como en los procesos.

Las PYME en el estado Zulia, específicamente las del sector de derivados lácteos, según Boscán (2005) presentan entre sus debilidades la insuficiente disponibilidad de recursos financieros, la escasez de tecnología de avanzada, carencia de recurso humano con formación y capacitación, asimismo, dificultad en el mantenimiento, reparación de maquinarias y la deficiente administración de costos y mercadeo, mientras que, entre las fortalezas destacan la calidad de sus productos, así como buenos canales de distribución y que utilizan insumos nacionales para sus procesos productivos.

Frente a estos planteamientos, el presente artículo tiene como objetivo fundamental analizar la utilización del crédito comercial como instrumento de financiamiento para las pequeñas y medianas empresas (PYME) productoras de derivados lácteos del estado Zulia, identificando adicionalmente las necesidades de financiamiento de este tipo de empresas. 
Con esta finalidad, se realizó una investigación descriptiva, de campo, no experimental y transversal, para lo cual se efectuó una entrevista, dirigida a los gerentes financieros de ocho (8) establecimientos dedicados a la producción y transformación de la leche cruda, ubicados en el estado Zulia, según las estadísticas proporcionadas por el Instituto de Investigaciones de la Facultad de Ciencias Económicas y Sociales de la Universidad del Zulia. Base de Datos del programa "Factores de competitividad de las pequeñas y medianas empresas (PYMES) de la región Zuliana”, 2004.

\section{Fuentes de financiamiento a corto plazo y aspectos teóricos sobre el crédito comercial}

La función de finanzas comprende tres decisiones fundamentales: inversión, financiamiento y dividendos (Van Horne, 1993). Dentro de este contexto el financiamiento representa una función de apoyo a las actividades primarias de la empresa, cuya finalidad es garantizar la disponibilidad oportuna de los recursos requeridos para el óptimo funcionamiento de éstas, tanto a corto como a largo plazo, logrando un equilibrio entre las necesidades de liquidez de la organización con sus objetivos de riesgo y rentabilidad (Vera, 2001).

Las fuentes de financiamiento a corto plazo están constituidas por todos aquellos pasivos que originalmente se hayan programado para liquidarse durante un periodo inferior a un año. Este tipo de financiamiento se utiliza para cubrir necesidades de fondos temporales, así como para brindar flexibilidad en un periodo de incertidumbre (Van Horne, 1993). Están conformadas por los pasivos acumulados como fuente interna y las cuentas por pagar (crédito comercial), los préstamos bancarios y papeles comerciales, como fuentes externas de financiamiento.

El funcionamiento de la actividad económica de una empresa puede financiarse con fondos propios de la misma o a través del crédito para su pago posterior. Por lo cual, en muchos casos el primordial pasivo circulante de las empresas son sus cuentas por pagar (pagos pendientes a otras empresas), constituyendo de este modo la principal fuente de financiación (financiamiento externo, a través de terceros: "Proveedores").

Weston y Copeland (1995), explican que el crédito comercial consiste en que una empresa compra a otras sus suministros y materiales sin pagar de contado registrando la deuda resultante como una cuenta por pagar. Frecuentemente este rubro constituye la categoría individual más grande de pasivos a corto plazo.

Según Bohórquez (2001), el crédito comercial, es una forma acostumbrada de hacer negocios en muchas industrias. Una organización que no califica para obtener crédito de una institución financiera, puede recibir el crédito a través de los proveedores, gracias a que la experiencia en las relaciones comerciales entre empresas favorece el surgimiento de confianza mutua así como el conocimiento de la capacidad de pago del cliente o comprador. De esta manera, el monto del crédito comercial fluctúa con las compras, de conformidad con los límites del mismo. 
Por otro lado, Van Horne (1993), señala las múltiples ventajas del crédito comercial como mecanismo de financiamiento a corto plazo, especialmente su fácil disponibilidad. Las cuentas por pagar de la mayor parte de las empresas representan una forma continua de crédito. No es necesario contratar un financiamiento formal. Si la empresa esta aprovechando los descuentos por pronto pago, puede disponer con facilidad de un crédito adicional, no pagando las cuentas existentes hasta el final del período concedido. No se requiere negociar con el proveedor, por cuanto la decisión depende por completo de ella. Por lo tanto, constituye una forma flexible de financiamiento, de carácter frecuentemente discrecional, aunque esta característica depende de la política respecto a la prontitud de pago que maneje cada empresa. La empresa no tiene que firmar un pagaré, ni comprometer colaterales o aceptar un programa de pagos estrictos en el pagaré.

El crédito comercial es un instrumento de financiamiento utilizado frecuentemente por las pequeñas y medianas empresas, debido a que los proveedores son más liberales al conceder créditos que las instituciones financieras; más aún en épocas donde los niveles de inflación presionan sobre el tipo de interés, lo que dificulta el aprovechamiento de préstamos bancarios dados los altos costos del financiamiento; en cambio para los proveedores la disminución de las ventas al contado provocada por la recesión económica impulsa la utilización del crédito comercial, bien sea por la vía de extender el plazo o aumentando sus cuentas por cobrar (Hernández, 2000). Sin embargo, en la decisión del uso de esta importante fuente de financiamiento, influyen las condiciones de crédito preestablecidas por parte del proveedor.

Según Block y Hirt (2001), el proveedor más grande de crédito a corto plazo usualmente está a las puertas de la empresa: el fabricante o vendedor de bienes y servicios. Aproximadamente, $40 \%$ de la financiación a corto plazo se presenta en la forma de cuentas por pagar o crédito comercial. Las cuentas por pagar son una fuente espontánea de fondos, que crece a medida que la empresa se expande sobre una base estacional o de largo plazo, y se contrae de una forma similar cuando el negocio decae.

El crédito comercial suele extenderse entre 30 y 60 días, muchas empresas tratan de ampliar el período de pago para recibir financiación adicional a corto plazo. Esa es una forma aceptable, en tanto no llegue a situaciones de abuso. Extender el plazo de 30 a 35 días puede ser tolerable dentro del comercio, mientras que llevarlo a 65 días podría molestar a los proveedores. Una variable importante para determinar el período de pago es la existencia de un descuento por compras de contado, de tal manera que a mayor descuento mayor probabilidad de reducir el tiempo de pago (Block y Hirt, 2001).

Como ya se dijo anteriormente, las empresas hacen compras a crédito a otros negocios y registran la deuda resultante como una cuenta por pagar. De allí que las cuentas por pagar, o crédito comercial constituya una deuda interempresarial que surge de las ventas a crédito, y que se registra como una cuenta por pagar para el comprador, al tiempo que representa un activo (cuenta por cobrar) 
para el vendedor. A partir de esta contrapartida, surge el concepto de "crédito comercial neto", entendido como un instrumento de financiamiento en el cual una empresa que realiza compras y ventas a crédito logra obtener una posición financiera favorable luego de cancelar las transacciones, es decir, si sus cuentas por cobrar son superiores a sus cuentas por pagar, en este caso la empresa esta en capacidad de extender un crédito comercial neto. Por el contrario, si las cuentas por pagar de una empresa exceden a sus cuentas por cobrar, se dirá que esta recibiendo un crédito comercial neto, de esta manera se convierte en un instrumento de financiamiento a corto plazo para la empresa receptora.

Frecuentemente, las empresas más grandes tienden a ser proveedores netos de crédito comercial (cuentas por cobrar relativamente altas), mientras que las empresas pequeñas se encuentran en la posición de usuarios, con cuentas por pagar relativamente altas (Block y Hirt, 2001).

La eficiencia en el manejo de las cuentas por pagar se mide a través del "periodo de diferimiento de las cuentas por pagar", el cual consiste en el plazo promedio de tiempo que transcurre entre la compra de los materiales y la mano de obra, y el pago en efectivo por los mismos (Weston y Brigham, 1996).

\section{$P D C P=\frac{\text { Cuentas por pagar }}{\text { Compras a crédito por día }}$}

donde PDCP es el período de diferimiento de las cuentas por pagar.

\section{El crédito comercial en las empresas del sector de derivados lácteos}

Al identificar las principales características de las empresas productoras de derivados lácteos del estado Zulia, se aprecia que, en su mayoría $(87,5 \%)$ constituyen empresas familiares, actualmente en la primera generación. Según el Decreto-Ley para la promoción de la pequeña y mediana empresa (República Bolivariana de Venezuela, 2001), en cuanto al número promedio de trabajadores anuales, pertenecen en un $62,5 \%$ de los casos analizados a la pequeña empresa, es decir, están conformadas por once (11) hasta cincuenta (50) personas, en tanto que, sólo el $12,5 \%$ corresponde a la mediana empresa, contratando entre cincuenta y un (51) hasta cien (100) personas, lo que evidencia que un $75 \%$ son PYME, el restante $25 \%$ corresponde a la microempresa, la cual le proporciona empleo a menos de 10 personas (Boscán et al., 2007).

En este sentido, se puede visualizar en el Gráfico 1, que las empresas productoras de derivados lácteos localizadas en el estado Zulia, en un $75 \%$ de los casos utilizan como primera alternativa de financiamiento las cuentas por pagar, que no es más que el crédito comercial o crédito con proveedores, corroborando la teoría que sostienen Weston y Copeland (1995), al afirmar que el primordial pasivo circulante de las empresas son sus cuentas por pagar (pagos pendientes a otras empresas), por la compra de suministros y materiales a crédito.

Por otro lado, el restante $25 \%$ de las empresas del sector, recurren a los préstamos bancarios, dado que los ban- 
cos suministran fondos para financiar necesidades estaciónales, expansión de la línea de producto y crecimiento a largo plazo. De este modo, se comparte la opinión de Hernández (2001), referida a que los préstamos de los bancos comerciales, ocupan el segundo lugar como fuente de financiamiento externa a corto plazo para las PYME (Gráfico 1).

Cabe destacar, que ninguno de los gerentes entrevistados ha utilizado los pasivos acumulados, a pesar de que es un tipo de deuda "gratuita", en el sentido de que no se paga ningún interés explícito sobre los fondos que se obtienen y representan una fuente espontánea de financiamiento a corto plazo. Esta situación podría explicarse, recordando que la mayoría de estas empresas son familiares (Boscán et al., 2007) y por tanto ocupan a personas integrantes del grupo familiar, por lo que es razonable inferir que no comprometerán ninguna de estas cuentas acumuladas.

Por otro lado, en cuanto a los criterios que emplean los gerentes de este tipo de industria, para seleccionar entre las diferentes alternativas de créditos a corto plazo figuran, con un $62,5 \%$ las condiciones del financiamiento, referidas a los montos, plazos, tasa de interés, requisitos, garantías, entre otros. Asimismo, en el $37,5 \%$ del total, el tiempo en que obtienen respuesta a la solicitud crediticia, es lo que principalmente ha motivado a los gerentes a escoger el financiamiento a corto plazo (Boscán y Sandrea, 2006).

Seguidamente, se analizará en detalle el uso de las cuentas por pagar como instrumento de crédito.

\section{Gráfico 1 \\ Fuentes de financiamiento \\ a corto plazo}

Préstamos Bancarios

$25,0 \%$

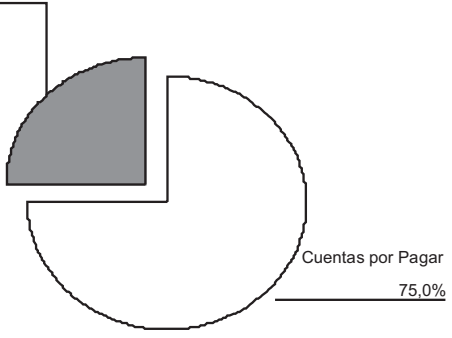

Fuente: Elaboración propia, con base en la encuesta realizada.

Según evidencias anteriores, el crédito comercial, constituye la principal fuente de financiamiento a corto plazo, utilizada por los gerentes del sector, debido a las facilidades para su otorgamiento en término de los requisitos exigidos y el período de aprobación del mismo.

Al respecto, es importante conocer cómo es la política de compra de las empresas estudiadas. Generalmente las compras que realizan las mismas pueden ser de contado, lo que significa cancelación inmediata; a crédito, considerando algunos términos o condiciones que rigen el otorgamiento del mismo; o mediante una combinación de ambas, es decir un porcentaje de las compras se realizan al contado y otro a través del financiamiento con otras empresas.

De acuerdo a los datos obtenidos en las empresas del sector de derivados lácteos, se determinó que el $62,5 \%$ del total de éstas, representada por cinco (5) de las ocho (8) investigadas, reflejaron que utilizan una combinación de compras 
a crédito y al contado, en tanto que el $25 \%$ manifestaron realizar sólo compras a crédito y solamente una (1) de las ocho (8) empresas, es decir el $12,5 \%$ realiza compras netamente al contado. Por lo que se puede afirmar que más del $80 \%$ realiza compras a crédito.

Por otra parte, en relación a los criterios considerados para la solicitud de un crédito comercial, el Gráfico 2 indica que el $62,5 \%$ de los gerentes entrevistados revelaron que el aspecto principal para solicitar un crédito comercial, es el plazo para la cancelación del mismo, que ofrecen los diversos proveedores, seguido de los descuentos por pronto pago con un $12,5 \%$, los nexos personales con los directivos de la empresa otro $12,5 \%$ y finalmente, una de las ocho empresas estudiadas no ofreció respuesta a este indicador (Gráfico 2).

Dado que el plazo para la cancelación del crédito, resultó ser el primordial motivo para solicitar un financiamiento externo con proveedores, se puede apreciar en el Gráfico 3, que del total de los gerentes entrevistados, el $50 \%$ manifestó que el plazo osciló entre 30 y 45 días, seguido de un $25 \%$ el cual indicó que el período para la cancelación del crédito exigido a su empresa fue menor a 30 días, sólo un $12,5 \%$ reveló que el plazo concedido a su empresa era mayor a 180 días y otro gerente $(12,5 \%)$ no ofreció información al respecto. Estos resultados se ajustan a la formulación hecha por Block y Hirt (2001), sobre los periodos promedios de pago y sus posibilidades de extensión en un margen tolerable (Gráfico 3).

\section{Gráfico 2 \\ Criterio para la solicitud del crédito comercial}

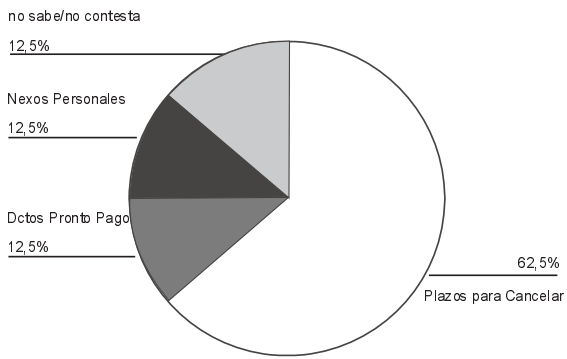

Fuente: Elaboración propia, con base en la encuesta realizada.

\section{Gráfico 3}

Plazo para la cancelación del crédito comercial

no sabe/no contesta

$12,5 \%$

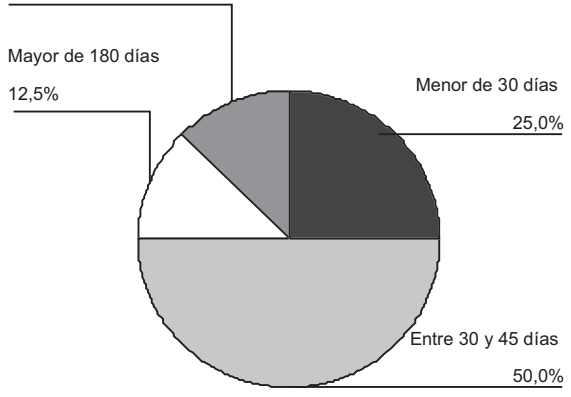

Fuente: Elaboración propia, con base en la encuesta realizada.

Una variable importante para determinar el período de pago es la posible existencia de un descuento por pronto pago, notando que en cuanto al porcentaje de descuento, el $75 \%$ del total de las empresas estudiadas, reciben entre $1 \%$ y $5 \%$ de reducción en el monto total del crédito, al cancelarlo antes de la fecha de pago, mientras que, un $25 \%$ no proporcionaron información al respecto. 
En cuanto al período de descuento, este osciló entre 5 y 10 días para tres de las ocho empresas, dos de ellas reciben entre 10 y 15 días de espera para la cancelación del crédito, una un período de 15 a 20 días y por último dos de los gerentes entrevistados no respondieron.

Paralelamente, al análisis de las cuentas por pagar de las empresas del sector de derivados lácteos del estado Zulia, también debe abordarse el estudio del financiamiento que estas ofrecen (cuentas por cobrar), por cuanto la diferencia entre estas dos cuentas dará como resultado el "crédito comercial neto".

En la Tabla 1, se determinan las compras a crédito diarias en unidades monetarias, revelando que el $37,5 \%$ de los gerentes de las empresas del sector realiza compras a crédito que oscilan entre 1 y 3 millones de bolívares, un $25 \%$ de 500.000 a 1 millón de bolívares, otro $25 \%$ menor a 500.000 bolívares y por último un $12,5 \%$ no respondió.

Por otro lado, al analizar las ventas a crédito diarias en unidades monetarias, se obtuvo como resultado que el $50 \%$ de las empresas del sector ofrece financiamiento, presentando unas cuentas por cobrar entre 3 y 5 millones de bolívares, en tanto que el $37,5 \%$ de 1 a 3 millones de bolívares y el restante $12,5 \%$ extiende un crédito de 500.000 a 1 millón de bolívares (Tabla 1).

Según Weston y Brigham (1996), si las cuentas por pagar de una empresa exceden a sus cuentas por cobrar, se dirá que está recibiendo un crédito comercial neto; si por el contrario las cuentas por cobrar exceden a sus cuentas por pagar, se estará extendiendo un crédito comercial neto.

Cabe destacar que, si se toma en cuenta particularmente cada uno de los montos establecidos, se encuentran diferencias notables, por cuanto las empresas en un $87,5 \%$ realizan compras a crédito que oscilan entre cero y tres millones diarios, mientras que un $50 \%$ realiza ventas a crédito por montos que fluctúan en ese mismo rango. Por lo tanto, dentro de esta escala de compra-venta, las empresas del sector de derivados lácteos, estarían recibiendo un crédito comercial neto, por cuanto sus cuentas por pagar superan las cuentas por cobrar. Sin embargo, analizando los datos obtenidos se puede visualizar que los empresarios realizan

Tabla 1

\section{Compras a crédito versus ventas a crédito diarias}

\begin{tabular}{lcc}
\hline & Compras \% & Ventas \% \\
\hline Menor a 500.000 Bs. & 25,0 & 0 \\
Entre 500.000 y 1 millón de Bs. & 25,0 & 12,5 \\
Entre 1 millón y 3 millones de Bs. & 37,5 & 37,5 \\
Entre 3 y 5 millones de Bs. & 0 & 50,0 \\
No sabe/no contesta & 12,5 & 0 \\
Total & 100,0 & 100,0 \\
\hline
\end{tabular}

Fuente: Elaboración propia, con base en la encuesta realizada. 
compras por un monto máximo de tres millones de bolívares, en tanto registran ventas por montos superiores de hasta cinco millones de bolívares diarios.

Ahora bien, al estudiar cada renglón por separado se puede apreciar que, de cero a un millón de bolívares, estarían recibiendo un crédito comercial neto. Entre uno y tres millones de Bolívares, las empresas ni extienden ni reciben un crédito comercial neto. No obstante, existe un $50 \%$ de las empresas que realiza ventas a crédito por un monto superior a los tres millones, al tiempo que ninguna de las empresas involucradas en el estudio efectúa compras a crédito por este último monto, por lo que en este rango se puede decir que las empresas otorgan un crédito comercial neto (cuentas por cobrar mayores que las cuentas por pagar) (Tabla 1).

Finalmente, a partir de esta información se podría inferir que las empresas del sector de derivados lácteos en general extienden un crédito comercial neto, evidenciado al examinar la opinión de los gerentes con respecto al crédito comercial neto, obteniendo como resultado que según un $75 \%$ de los consultados el crédito comercial otorgado a su empresa nunca supera el nivel de las cuentas por cobrar, en tanto que sólo un $12,5 \%$ representado por un gerente, reveló que alguna vez las cuentas por cobrar de su empresa fueron inferior a las cuentas por pagar y el restante $12,5 \%$ no reveló información al respecto (Gráfico 4).

Lo anterior contrasta con la teoría de Block y Hirt (2001), quienes sostienen que frecuentemente las empresas pequeñas se encuentran en la posición de usuarios, con cuentas por pagar relativamente altas.

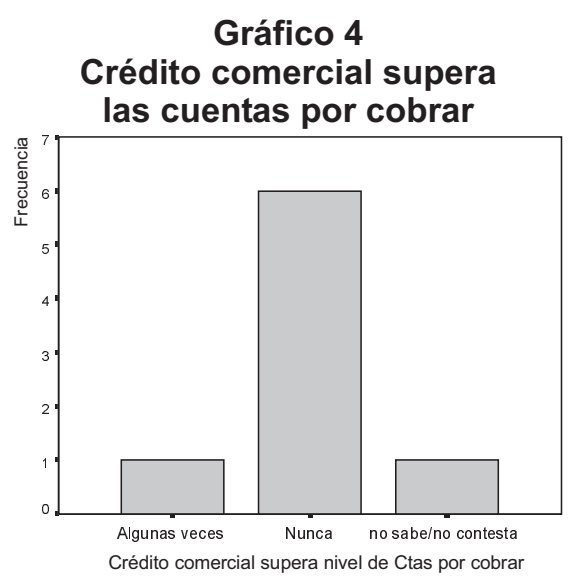

Fuente: Elaboración propia, con base en la encuesta realizada.

\section{Necesidades de financiamiento en las empresas del sector de derivados lácteos del estado Zulia}

Luego del diagnóstico acerca del uso del crédito comercial como fuente de financiamiento de corto plazo, se consideró, dado la evidente necesidad de recursos monetarios que éstas empresas requieren, indagar sobre las necesidades de financiamiento del sector. En este orden de ideas, se analizaron los siguientes indicadores: destino del crédito, monto, plazos y tasa de interés.

\subsection{Destino del crédito}

Al analizar el indicador destino del crédito, se obtuvo que el $37,5 \%$ del total de las empresas, utilizarían los préstamos para cancelar obligaciones relacionadas con el capital de trabajo, en las que destacan la compra de materias primas, materiales, inventarios, entre otros; otro 
$37,5 \%$ opinaron que los emplearían específicamente para la adquisición de maquinarias y equipos, es decir, aumento de capacidad instalada; y un $25 \%$ en ampliación de la infraestructura. Todos los créditos, en opinión de los gerentes entrevistados, están dirigidos al crecimiento del negocio, en las modalidades ya mencionadas (Gráfico 5).

\subsection{Monto del financiamiento.}

El $87,5 \%$ representado por siete de las empresas estudiadas, reflejaron que el importe del préstamo requerido por la industria productora de derivados lácteos, para financiar el crecimiento del negocio u otras obligaciones supera los cincuenta (50) millones de bolívares por empresa, en tanto que para el $12,5 \%$ puede oscilar entre 5 y 10 millones de bolívares. Este resultado tiene su explicación en el hecho que este tipo de empresas necesita maquinarias y equipos importados, por lo que dependiendo de la tecnología que deseen adquirir el monto pudiera ser cada vez mayor (Gráfico 6).

\subsection{Plazo de cancelación}

El análisis de este indicador, permitió detectar que las empresas de derivados lácteos ubicadas en el estado Zulia, requieren un período de tiempo que fluctúe entre 1 y 3 años, para la cancelación del crédito, lo que representa el $62,5 \%$ de las opiniones, un $25 \%$ de estas lo requieren para pagarlo en más de 3 años y el porcentaje más bajo un $12,5 \%$ podría cancelarlo en un período menor o igual a 1 año (Gráfico 7).

\section{Gráfico 5 \\ Destino del crédito obtenido por las empresas del sector}

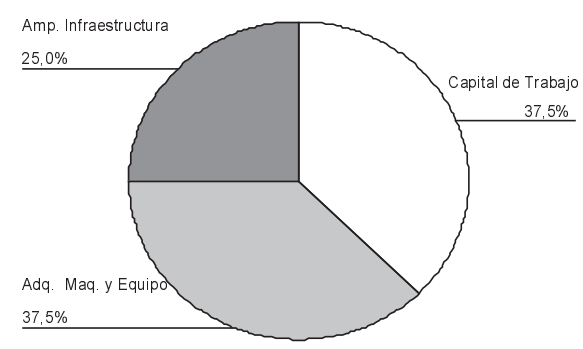

Fuente: Elaboración propia, con base en la encuesta realizada.

\section{Gráfico 6 \\ Monto del financiamiento requerido por las empresas del sector}



Fuente: Elaboración propia, con base en la encuesta realizada.

\subsection{Tasa de interés}

Los resultados obtenidos revelaron, que la tasa de interés que soportarían o estarían en capacidad de pagar todas las empresas del sector oscila entre $10 \%$ y $20 \%$. Por lo que, si se tiene como objetivo tratar de relanzar o reactivar el 


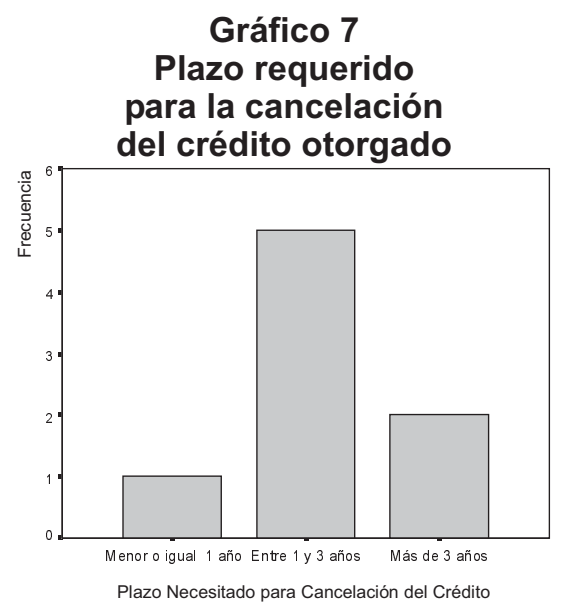

Fuente: Elaboración propia, con base en la encuesta realizada.

papel de este tipo de empresas a nivel regional, es importante ofrecer tasas de interés atractivas.

\section{Conclusiones}

De las fuentes de financiamiento a corto plazo manejadas por las empresas del sector de derivados lácteos, el crédito comercial conforma el instrumento de financiamiento más utilizado, por cuanto las condiciones de este tipo de crédito referidas a los montos, plazos, tasa de interés, requisitos, garantías, entre otros, figuran como primordial opción entre los criterios que emplean los gerentes al momento de seleccionar entre las diferentes alternativas de financiamiento.

La mayoría de las empresas, reflejaron que utilizan una combinación de compras a crédito y al contado; además el criterio mayormente considerado para la solicitud de un crédito comercial, es el plazo para la cancelación. Asimismo, los empresarios afirman que el crédito comercial otorgado a su empresa nunca supera el nivel de las cuentas por cobrar, por lo que estas extienden un crédito comercial neto.

Al analizar las necesidades de financiamiento a corto plazo de las empresas consideradas, se encontró que el destino de los recursos monetarios, esta relacionado básicamente con el capital de trabajo (materias primas, materiales, inventarios, vehículos, entre otros). De igual forma, algunos lo emplearía en la adquisición de maquinarias y equipos, considerados vital para la elaboración de sus productos y crecimiento del negocio y una minoría lo orientaría a la ampliación de la infraestructura.

Por último, este tipo de empresas considera que el importe para financiar el crecimiento de sus negocio $u$ otras obligaciones supera los cincuenta (50) millones de Bolívares; requiriendo un período de tiempo para la cancelación del crédito, que fluctúe entre 1 y 3 años, estando en capacidad de pagar todas las empresas del sector una tasa de interés que oscile entre $10 \%$ y $20 \%$. Todas estas condiciones proporcionan una buena orientación para el diseño de políticas de financiamiento de carácter tanto público como privado, si se tiene como objetivo tratar de relanzar o reactivar el papel de este tipo de empresas a nivel regional.

\section{Referencias bibliográficas}

\footnotetext{
Block, Stanley y Hirt, Geoffrey (2001). Fundamentos de Gerencia Financiera. Editorial McGraw-Hill Interamericana S.A., Bogotá, Colombia. Pp. 580.
} 
Bohórquez, Elizabeth (2001). Estrategias Financieras Aplicadas en las Industrias del Sector Lácteo en la Región Zuliana. Año 2001. Tesis de Grado para optar al Título de Magíster en Gerencia de Empresas. Universidad del Zulia, Maracaibo, Venezuela.

Boscán, Mariby (2005). Fuentes de financiamiento utilizadas por las pequeñas y medianas industrias (PYMI) productoras de derivados lácteos en el estado Zulia. Tesis de Grado para optar al Título de Magíster en Gerencia de Empresas. Universidad del Zulia, Maracaibo, Venezuela.

Boscán, Mariby y Sandrea, Maryana (2006). Estrategias de financiamiento para la incorporación de innovaciones tecnológicas impulsoras del desarrollo endógeno en el sector lácteo. Revista Estudios Iberoamericanos. Año 1, No. 1, Primer semestre, Venezuela. Instituto de Estudios iberoamericanos, pp. 9-28

Boscán, Mariby; Romero, Jenny y Sandrea, Maryana (2007). Fuentes de financiamiento utilizadas por las empresas productoras de derivados lácteos del estado Zulia. Revista de Ciencias Sociales (RCS). Vol. XIII, No. 1, Venezuela. Instituto de Investigaciones, Universidad del Zulia, pp. 134-146

Hernández, Lissette (2000). El Financiamiento de la Pequeña y Mediana Industria (PYMI) en la Región Zuliana (Sector Confección, 1998-1999). Tesis de Grado para optar al Título de Magíster en Gerencia de Empresas. Universidad del Zulia, Maracaibo, Venezuela.

Hernández, Lissette; Romero, Jenny; Portillo, Rafael; Hernandez, Ridelis (2001).
Las fuentes de financiamiento a corto plazo y su administración en la pequeña y mediana industria (pymi) en la región zuliana. (Sector confección, 1998-1999). Revista Ciencias Sociales (RCS), Vol. VII No. 1, Venezuela. Instituto de Investigaciones, Universidad del Zulia, pp. 112-137.

Instituto de Estudios Superiores de Administración (IESA) (1997). Zulia: Competitiva para el Desarrollo. Ediciones IESA, C.A, Caracas.

Instituto de Investigaciones (2004). Base de datos programa "Factores de competitividad de las Pequeñas y Medianas empresas manufactureras en la región zuliana". Maracaibo, Venezuela. Mimeo.

República Bolivariana de Venezuela (2001). Decreto Ley para la promoción y desarrollo de la pequeña y mediana industria. Gaceta Oficial. Caracas. 12-11-2001. No. 5.552 extraordinario.

Van horne, James (1993). Administración Financiera. Editorial Prentice Hall Hispanoamericana, S.A., México, Distrito Federal.

Vera, Mary (2001). Gestión Financiera de la Pequeña y Mediana Industria en la ciudad de Maracaibo. Revista Ciencias Sociales (RCS), Vol. VII, No. 1, Venezuela. Instituto de Investigaciones, Universidad del Zulia, pp. 65-89.

Weston, F. y Brigham, E. (1996). Fundamentos de Administración Financiera. Editorial McGraw-Hill, México, Distrito Federal.

Weston, F. y Copeland, T. (1995). Finanzas en Administración. Volumen II. Editorial McGraw-Hill, México, Distrito Federal. 